\title{
Non-adherence to Antiretroviral Treatment Episodes and Contributing Factors among Fully Disclosed Adolescents with Perinatal HIV in the Kingdom of Lesotho
}

\author{
Ntaoleng Mohlabane, Sphiwe Madiba* \\ Department of Public Health, School of Health Care Sciences, Sefako Makgatho Health Sciences University, \\ Pretoria 0001, South Africa
}

Received July 8, 2021; Revised September 9, 2021; Accepted October 17, 2021

\section{Cite This Paper in the following Citation Styles}

(a): [1] Ntaoleng Mohlabane, Sphiwe Madiba, "Non-adherence to Antiretroviral Treatment Episodes and Contributing Factors among Fully Disclosed Adolescents with Perinatal HIV in the Kingdom of Lesotho," Universal Journal of Public Health, Vol. 9, No. 5, pp. 332 - 338, 2021. DOI: 10.13189/ujph.2021.090516.

(b): Ntaoleng Mohlabane, Sphiwe Madiba (2021). Non-adherence to Antiretroviral Treatment Episodes and Contributing Factors among Fully Disclosed Adolescents with Perinatal HIV in the Kingdom of Lesotho. Universal Journal of Public Health, 9(5), 332 - 338. DOI: 10.13189/ujph.2021.090516.

Copyright@2021 by authors, all rights reserved. Authors agree that this article remains permanently open access under the terms of the Creative Commons Attribution License 4.0 International License

\begin{abstract}
Children and adolescents with Perinatal Acquired HIV (PHIV) face many challenges to remain adherent to long-term Antiretroviral Therapy (ART). Among adolescents, adherence is often not static but changes over time due to the circumstances of the individual. The study assessed the ART adherence levels and patterns of adolescents with PHIV who were receiving long-term ART. This quantitative cross-sectional study was conducted in Mohale's Hoek, a rural Health District in Lesotho between May and December 2018. Adolescents aged 10-19 years who were aware of their HIV status were recruited from selected youth-friendly HIV clinics. Adherence was measured through a self-report of the last pills missed, based on 30 days recall. Stata version 13 was used for analysis. The sample consisted of 130 adolescents, $57 \%$ were females, the mean age was 15 years, the mean age of ART initiation was nine years, and the mean ART duration was four years. Self-reported adherence was very high at $92 \%$, and $88 \%$ reported not delaying taking ART. Self-reported optimal ART adherence was achieved by $85 \%$ of the adolescents, $15 \%$ were non-adherent, and 92.3\% had not missed clinic appointments in the past 30 days. Episodes of non-adherence among those who delayed taking ART on time occurred in the evenings, mornings, weekdays, and weekends. Non-adherence episodes for
\end{abstract}

$59 \%$ occurred more in the evenings than other periods of the day. Conclusion: The results suggest that despite high self-reported adherence, adolescents take medication inconsistently and have situational adherence patterns. The dosing of medication and regimens should be flexible and take into consideration the context of the adolescents.

Keywords Adolescents, ART Adherence, Disclosure, Lesotho, Perinatally Acquired HIV, Self-Reports

\section{Introduction}

With the success of Antiretroviral Therapy (ART), there has been an increasing number of children with Perinatal HIV (PHIV) who are maturing into adolescents and young adults [1]. As such, children and adolescents with PHIV are a growing population in sub-Saharan Africa [2]. Adherence to the ART regimen remains one of the important concerns when providing ART to children and adolescents with PHIV. This is particularly important in resource-limited countries where second-and third-line regimens are not readily available and could eliminate children's limited treatment options [3,4]. 
Good adherence is defined as following the recommendations made by treatment providers or healthcare workers in agreement with the patient on timing, dosage, and frequency of medication taking [5]. Good adherence is measured as $\geq 95 \%$ in pill counts; it also relies heavily on patients' recall of the days in which they missed doses and pharmacy prescription refills. Although self-reported measures remain the most commonly used method to determine adherence in sub-Saharan Africa [6], the gold standard for assessment of treatment adherence is the viral load measurement [3]. The viral load measure is a cardinal marker of how well an individual is doing on ART and adherence to the ART regimen [7].

Adolescents face unique medication dosing problems due to rapid growth and puberty and experience HIV-related stigma [8]. The goal of ART is to achieve viral suppression to reduce AIDS-related morbidity and mortality due to HIV [9], and medication adherence is critical to achieving this goal. When compared with younger children and adults, adolescents are the only age group where HIV-related rebound mortality, serious illness leading to long-term hospitalization, and comparatively poor treatment responses are increasing $[1,10,11,12]$.

To have long-term success on ART implies that the correct dose of appropriate ART is being consistently and correctly taken [13]. Research suggests that non-adherence is often not static but changes over time due to the circumstances of the individual [8]. The patterns of adherence and perceptions towards ART change during the life course of individuals on long-term ART [10]. For some adolescents non-adherence behavior changes when they mature and take responsibility for their treatment or when they are changed to a different ART regimen $[1,2]$

Adolescents with PHIV have different adherence patterns which are often situational [1,14]. Situational adherence or episodic adherence is when an individual, including adolescents with PHIV, takes medication inconsistently. Hawkins et al [1] describe situational adherence patterns as having a period of adherence and non-adherence within a short period influenced largely by factors such as location at the time of dosing, or the time of day when the individual's medication is due. For example, school activities often outweigh clinic appointments and medication refills, which result in non-adherence for a short duration [1,15]. Episodic adherence described as the missed or late doses, treatment interruptions, and discontinuations, and partial dosing leads to suboptimal ART adherence [16].

In Lesotho, clinical data show that a significant proportion of adolescents have been switched from the first to the second or and third line of ART drug regimens because of poor adherence. Clinical data from the youth-friendly clinics suggest that adolescents attending
Network Clubs or Teen Clubs have improved adherence. The Teen Clubs offer HIV and ART adolescent-friendly services to address adherence and retention in care of adolescents with PHIV [17]. However, adolescents with PHIV have been associated with different and often situational non-adherence patterns [1,14]. Against this backdrop, this study assessed the ART adherence pattern of adolescents attending Network Clubs in Lesotho using self-reports. Understanding adherence patterns in this population can strengthen the psychosocial support interventions provided to adolescents through the Network Clubs. Services must provide comprehensive HIV services and strengthen social support and coping strategies to support adolescents adhere to their ART, despite the presence of HIV-related stigma [18].

\section{Materials and Methods}

This paper presents data from a larger cross-sectional study that was conducted in nine adolescent-friendly clinics in the Mohale's Hoek district of the Kingdom of Lesotho [19]. The Kingdom of Lesotho is a landlocked country surrounded by South Africa. Mohale's Hoek Health District, where the study setting was located is predominantly rural and mountainous. The district is located in the southern region of the Kingdom and shares borders with South Africa. It has 15 health facilities, of which three are private clinics. The clinics run Network Clubs and offer a peer-support program providing adolescents with counseling, addresses HIV knowledge, sexual and reproductive (SRH) education, adherence, disclosure, the acceptance of HIV status, and other psychosocial issues $[17,20]$

\subsection{Study Population}

The study participants were all children and adolescents aged between 10-19 years who were receiving medical services and ART from the selected facilities. Approximately 800 children and adolescents were enrolled in these facilities, at the time of the data collection. Of these, 130 adolescents with PHIV aged between 10 and 19 years were recruited using the convenience sampling method. Permission to access the health facilities was obtained from the District Health Management Team in Mohale's Hoek Health District. The first author who was responsible for data collection, met with the nurse managers of the health facilities to request permission to conduct the study in their facilities. The nurse clinicians facilitated the introductions between the caregivers, the adolescents, and the researcher team. In addition, the nurse clinicians identified adolescents who met the inclusion criteria and referred them to the research team. Participants were eligible to participate in the study if they had been fully disclosed to, were aware of their 
HIV status, and had attended three or more Network Club sessions at the time of the data collection. Knowing their HIV status was a requirement for enrolment in the Network Clubs.

\subsection{Data Collection}

A researcher-administered quantitative validated tool adapted from the AIDS Clinical Trials Group [21] was used to measure the level of adherence to ART. The tool from the AIDS Clinical Trial Group also asked questions on sexual behavior which were excluded in the current study. The questions were divided into three sections: (i) socio-demographic characteristics, (ii) clinical information, and (iii) adherence questions. Clinical information included questions such as the duration of ART, the ART start date, and the number of pills. Adherence was measured through self-report of the last pills missed, based on the 30 days and seven days recall of the pills taken (validated through the medical records). The tool was structured, translated into Sesotho, and researcher-administered. We opted to administer the tool instead of requesting the adolescents to complete the tool themselves. Some were aged as young as 10 years. The first author of this paper and a trained research assistant collected data on the days when the Network Club sessions were offered.

Adolescents under the age of 18 years were interviewed only after obtaining caregivers' written informed consent and their written assent. Written, informed consent was obtained from adolescents 18 years old and above. Informed consent and assent were administered in Sesotho. The questionnaire was completed in a private room in the absence of the parent/caregiver for those who had been accompanied to the clinic. The completed questionnaires were kept in a locked cupboard, and only the authors had access to the data.

\subsection{Ethical Considerations}

Ethical approval was sought from the Research Ethics Committee of Sefako Makgatho Health Sciences University [SMUREC/H/257/2017: PG] and permission from the Ministry of Health, Lesotho [REF: ID: 46-2018] and the District Health Management Team. Participation was voluntary and adolescents younger than 18 years signed an assent form and their caregivers signed informed consent. Those 18 years and older signed their informed consent.

\subsection{Data Analysis}

Descriptive statistics were used to summarise the socio-demographic characteristics, the clinical characteristics, and the levels of adherence of the participants. All categorical variables were reported as absolute numbers and percentages. The means of continuous variables like age and the duration on ART were computed using the student t-test.

\section{Results}

\subsection{Characteristic of the Participants}

The characteristics of the participants are described in Table 1. All were at the clinic for their ART refills, the majority (59\%) were 15 years and younger, the median age of the participants was 15 years (range $10-19$ years). Over half $(56 \%)$ were female, $84.6 \%$ came to the clinic alone, and $82.3 \%$ were currently in school. The majority (75\%) used public transport to get to their nearest clinic and paid an average of R14.00 (\$0.89) per trip. More than a quarter $(37.7 \%)$ of the adolescents were maternal orphans, $58.5 \%$ reported their fathers deceased, and $24.6 \%$ were categorized as double orphans. Most $(41.5 \%)$ of them were living with their grandparents. The majority $(100 / 76.9 \%)$ reported that their primary caregivers were unemployed.

Table 1. Characteristics of the study participants $(n=130)$

\begin{tabular}{|c|c|}
\hline Variable/Categories & Freq/Percent \\
\hline \multicolumn{2}{|l|}{ Sex } \\
\hline Male & $56(43)$ \\
\hline Female & $74(57)$ \\
\hline \multicolumn{2}{|l|}{ Age category } \\
\hline$<15$ years & $77(59)$ \\
\hline$>15$ years & $53(41)$ \\
\hline \multicolumn{2}{|l|}{ Attending school } \\
\hline No & $23(17.7)$ \\
\hline Yes & $107(82.3)$ \\
\hline \multicolumn{2}{|l|}{ Walk to the clinic } \\
\hline No & $98(75.4)$ \\
\hline Yes & $49(24.6)$ \\
\hline \multicolumn{2}{|l|}{ Primary caregiver } \\
\hline Both parents & $13(10)$ \\
\hline Grandparents & $26(26)$ \\
\hline Mother & $9(6.9)$ \\
\hline Father & $54(41) .5$ \\
\hline Guardians & $17(13) 1$. \\
\hline Siblings & $9(6.9)$ \\
\hline Alone & $2(1.5)$ \\
\hline \multicolumn{2}{|l|}{ Caregiver employment status } \\
\hline Employed & $25(19.2)$ \\
\hline Unemployed & $100(76.9)$ \\
\hline Pensioner & $5(3.9)$ \\
\hline \multicolumn{2}{|l|}{ Maternal orphan } \\
\hline Yes & $49(37.7)$ \\
\hline No & $81(62.3)$ \\
\hline \multicolumn{2}{|l|}{ Paternal orphan } \\
\hline Yes & $76(59)$ \\
\hline No & $53(41)$ \\
\hline
\end{tabular}




\subsection{ART Characteristics}

The average number of tablets taken per day was two (range 1-9) and a significant proportion $(n=26,20 \%)$ of the participants were taking more than three tablets per day. The majority $(n=50,38.5 \%)$ had started taking ART between the ages of 11 and 15 years and the mean age of ART initiation was 9 years $(S D=5.8)$. Almost half $(n=63$, $48.5 \%$ ) had been receiving ART for not more than five years, the median time receiving ART was 4 years $(\mathrm{SD}=$ 4.19), $15 \%$ of the adolescents did not know when they had started ART, possibly because they had been receiving ART since birth (table 2).

\subsection{Short Term Adherence Recall}

Adherence was measured through self-report of last missed pills based on the 30 days recall of pills taken, optimal adherence was measured as ingesting $>95 \%$ of doses per month as prescribed. The majority $(n=92,85 \%)$ reported that they had not missed a single dose in the past 30 days (Figure 1). There were no significant differences by age groups, gender, living arrangement, and duration on ART for achieving optimal adherence. The majority $(92 \%, 120)$ had not missed an ART clinic appointment in the past 30 days. Only $10(7.6 \%)$ reported having missed an appointment. Of those that missed a clinic appointment, the common cited reasons were not having money for transport $(n=4)$, being away from home $(n=4)$, at school with exams $(n=1)$ and simply forgot $(n=1)$.

Table 2. ART and clinical characteristics

\begin{tabular}{|c|c|}
\hline Variable Category & Frequency \\
\hline \multicolumn{2}{|l|}{ Age ART initiation } \\
\hline $1-5$ years & $17(13.1)$ \\
\hline $6-10$ years & $26(20)$ \\
\hline $11-15$ years & $50(38.5)$ \\
\hline $16-19$ years & $17(13.1)$ \\
\hline Don't know & $20(15.4)$ \\
\hline \multicolumn{2}{|l|}{ Time on ART } \\
\hline$<1$ year & $11(8.5)$ \\
\hline $1-5$ years & $63(48.5)$ \\
\hline $6-10$ years & $25(19.3)$ \\
\hline ears & $11(8.5)$ \\
\hline$>15$ years & $3(2.3)$ \\
\hline Don't know & $17(13.1)$ \\
\hline \multicolumn{2}{|l|}{ Number of tablets } \\
\hline 1 tablet & $35(26.9)$ \\
\hline 2 tablets & $48(36.9)$ \\
\hline 3 tables & $21(21.2)$ \\
\hline$<3$ tables & $26(20)$ \\
\hline
\end{tabular}

\section{Missed taking ART past 30 days}

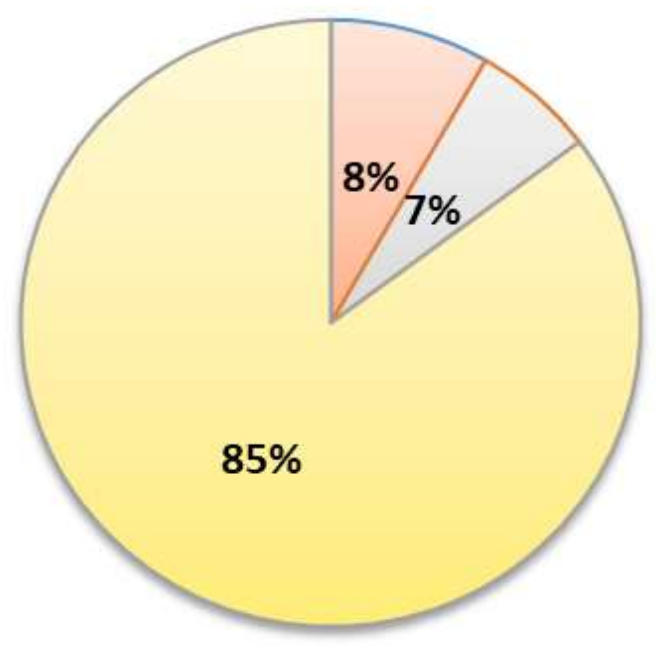

\section{1-2weeks ago $\square$ 2-4weeks ago $\square$ none}

Figure 1. Self-reported number of missed doses in past 30 days 


\subsection{Self-reported Adherence to ART}

To assess adherence to ART in terms of time, dose, and appointments, the participants were asked if they ever encountered problems taking ART on time. The majority $(\mathrm{n}=119,91.5 \%)$ reported that they did not delay taking ART on time while $16(21 \%)$ said they delayed taking ART on time. Slightly more females (10) than males (6) delayed taking ART on time, and those $<15$ years (11) delayed taking ART slightly more than those $>15$ years (5) did. Concerning the period of day and week or weekend when they had problems keeping time, over half $(n=76$, $58.5 \%)$ had a problem keeping time in the evenings and $14(10.8 \%)$ had a problem keeping time in the mornings, 6 (4.6\%) had problems keeping time on weekdays and weekends respectively (Table 3 ).

Table 3. Adherence patterns and non-adherence episodes

\begin{tabular}{|c|c|c|}
\hline Variable statement & Frequency & Percentage \\
\hline Delayed taking ART & 16 & 12 \\
\hline Did not delay taking ART & 113 & 88 \\
\hline Non-adherent episodes & & \\
\hline Evenings & 76 & 59 \\
\hline Mornings & 14 & 11 \\
\hline All the time & 1 & 1 \\
\hline Weekdays & 6 & 5 \\
\hline Weekends & 6 & 5 \\
\hline None & 27 & 21 \\
\hline
\end{tabular}

\section{Discussion}

The study assessed ART adherence levels and patterns among adolescents using self-reported questionnaires. All the participants had been disclosed to fully and were attending Network Clubs as standard care for adolescents with PHIV in Lesotho to improve adherence. While the WHO recommends that children be disclosed their HIV status by 10-12 years old, the mean age of enrolment in care of the adolescents in this study was 15 years, suggesting that they were unaware of their HIV status until late adolescence. Other studies in sub-Saharan Africa reported on the late diagnosis of HIV and late enrolment in care in adolescents with PHIV [17,20,22]. The study found that $20 \%$ of the adolescents were taking more than three tablets per day; the mean duration on ART was four years.

We found that self-reported adherence was high; the optimal ART adherence (>95\%) was achieved by $85 \%$ of the adolescents based on the 30 days recall of the last pills missed; only $15 \%$ reporting missing ART in the past month and were classified as non-adherent. In a recent study conducted in Cameroon, the self-report measure of adherence was achieved by $83 \%$ of adolescents. This was also based on 30 days recall of the last pills taken [23]. Regarding missing clinic appointments, 92.3\% had not missed an appointment in the past 30 days. Although the majority of participants reported that they had not missed doses in the past 30 days, we found that similar to what has been described by Hawkins et al [1] as situational adherence patterns; the adolescents had episodes of non-adherence. Situational adherence is described as having a period of adherence and non-adherence within a short period influenced largely by factors such as location at the time of dosing, or the time of day, an individual is due to take medication. In the current study, almost a quarter $(21 \%)$ of the adolescents delayed taking ART at the scheduled time.

The study found that most episodes of non-adherence among those who delayed taking ART on time occurred in the evenings. Almost two-thirds (59\%) had problems keeping to the time of ART in the evening. This might be because the drug regimen for the majority of the adolescents contains Efavirenz and is taken in the evenings. Since the majority of adolescents were on the second regimen or Lopinavir-based ART regimen, they could not benefit from the once-daily fixed-dose combination regimens which have served as a strategy to address non-adherence in other settings [6].

In the current study, non-adherence episodes in the evenings were due to conflicting activities at the time of medication such as late study time mentioned by those at boarding schools. Similarly, research suggests that the reason for the delay might have been the situation or the context of the adolescents at the time of medication $[1,3,24]$. In a study conducted in Botswana, Madiba and Josiah [24] found that any disruptions in the routine dosing time such as after-school activities and sporting events which kept the adolescents from their homes affected adherence. Therefore, treatment schedules must link to realistic daily activities of adolescents such as strategies to maintain adherence while away from home [6]. Health care professionals should routinely assess short-term adherence to identify episodic adherence to obtain more accurate measures for ART adherence among adolescents with PHIV [25].

The study did not find that non-adherence episodes occurred more over weekends as reported in other studies [1]. Adolescents in the current study attend Network Club sessions on weekends, and over a third reported that they collect their medications on weekends only. Furthermore, consistent with previous studies, there was no statistically significant association between gender and non-adherence episodes [16,24].

The living arrangements of children and adolescents with PHIV have a bearing on their ability to sustain adherence to long-term ART regimens and keeping clinic appointments [24]. The home is the main place where adolescents take their medication to manage their disease [3]. In the current study, $41.5 \%$ of the adolescents were 
cared for by their grandparents, and $72 \%$ of those who reported to have problems taking ART on time were cared for by their grandparents. Grandparents have been associated with suboptimal adherence in adolescents as they often forget clinic appointments and taking medication on time [13]. Other studies reported that adolescents who have both parents living with them were more adhering as compared to those living with single caregivers [22,23].

\section{Limitations}

A major limitation of the study is that we used self-reports that can only assess short-term adherence. Self-reports are subject to social desirability and often participants overestimate adherence. Secondly, self-reports are based on recall that is subject to recall bias in providing adherence behavior. However, we asked additional questions about the times when participants had problems taking medication on time, and this yielded higher accuracy for identifying non-adherence than asking participants whether they were missing doses [25].

\section{Conclusions}

The study found good adherence based on self-reports as well as high non-adherence episodes occurring in the evenings more than other periods of the day. The dosing of medication and regimens should be flexible and take into consideration the context of the adolescents, given that the adherence episodes are dynamic and changing over time and may differ among older and younger adolescents. Therefore, interventions such as adherence counseling provided through the Network Clubs should be tailored to the specific needs of this group and include their caregivers.

It is crucial that intervention strategies to mitigate against adherence barriers focus on situational adherence issues such as the ones highlighted in the current study. There is a need to strengthen adolescent-friendly services and training of healthcare workers in these services in Lesotho and other settings with similar contexts.

\section{REFERENCES}

[1] Hawkins A., Evangeli M., Sturgeon K., Le Prevost M., Judd A., AS. Committee, "Episodic medication adherence in adolescents and young adults with perinatally acquired HIV: a within-participants approach," AIDS Care, vol. 28, no. (sup1), pp. 68-75, 2016. DOI: $10.1080 / 09540121.2016 .1146210$

[2] Adejumo OA., Malee KM., Ryscavage P., Hunter SJ., BO. Taiwo, "Contemporary issues on the epidemiology and antiretroviral adherence of HIV - infected adolescents in sub - Saharan Africa: a narrative review," Journal of the International AIDS Society, vol. 18, no. 1, pp. e20049, 2015. DOI: 10.7448/IAS.18.1.20049

[3] Denison JA., Banda H., Dennis AC., Packer C., Nyambe N., Stalter RM., Mwansa JK., Katayamoyo P., DR. McCarraher, "The sky is the limit": adhering to antiretroviral therapy and HIV self-management from the perspectives of adolescents living with HIV and their adult caregivers," Journal of the International AIDS Society, vol.18, no.1, pp. e19358, 2015. DOI: 10.7448/IAS.18.1.20049

[4] Mutwa PR., Van Nuil JI., Asiimwe-Kateera B., Kestelyn E., Vyankandondera J., Pool R., Ruhirimbura, J., Kanakuze, C., Reiss, P., Geelen, S., JVD. Wijgert, "Living situation affects adherence to combination antiretroviral therapy in HIV-infected adolescents in Rwanda: a qualitative study," PLoS One, vol.8, no. 4, pp. e60073, 2013. DOI: 10.1371/journal.pone.0060073

[5] Bukenya D., Mayanja BN., Nakamanya S., Muhumuza R., J. Seeley, "What causes non-adherence among some individuals on long term antiretroviral therapy? Experiences of individuals with poor viral suppression in Uganda," AIDS Research and Therapy, vol. 16, no. 1, pp.1-9, 2019. DOI: 10.1186/s12981-018-0214-y

[6] Kim MH., Mazenga AC., Yu X., Ahmed S., Paul ME., Kazembe PN., EJ. Abrams, "High self - reported non adherence to antiretroviral therapy amongst adolescents living with HIV in Malawi: barriers and associated factors," Journal of the International AIDS Society, vol. 20, no. 1, pp. e21437, 2017. DOI: 10.7448/IAS.20.1.21437

[7] Gokarn A., Narkhede MG., Pardeshi GS., MK. Doibale, Adherence to antiretroviral therapy. Journal of the Association of Physicians of India, vol. 60, pp. 16-20. 2012.

[8] Agwu AL., L. Fairlie, “Antiretroviral treatment, management challenges and outcomes in perinatally HIV infected adolescents," Journal of the International AIDS Society, vol.16, no. 1, pp. e18579, 2013. DOI: 10.7448/IAS.16.1.18579

[9] The Joint United Nations Programme on HIV/AIDS (UNAIDS). Ending AIDS: progress towards the 90-90-90 targets. 2017. Available from; http://www.unaids.org/sites/ default/files/media_asset/global_aids update_2017_en.pdf. 2017 (Assessed November 2020)

[10] Nglazi MD., Kranzer K., Holele P., Kaplan R., Mark D., Jaspan H., Lawn SD., Wood R., LG. Bekker, "Treatment outcomes in HIV-infected adolescents attending a community-based antiretroviral therapy clinic in South Africa," BMC Infectious Diseases, vol. 12, no. 1, pp. 1-7, 2012. DOI: 10.1089/aid.2012.0215

[11] Ankrah DN., Koster ES., Mantel-Teeuwisse AK., Arhinful DK., Agyepong IA., M. Lartey, "Facilitators and barriers to antiretroviral therapy adherence among adolescents in Ghana," Patient Preference and Adherence, vol. 10, pp. 329-337. 2016. DOI: 10.2147/PPA.S96691

[12] Inzaule SC., Hamers RL., Kityo C., de Wit TFR., M. Roura, "Long-Term Antiretroviral Treatment Adherence in HIV-Infected Adolescents and Adults in Uganda: A Qualitative Study,” PLoS One, vol.11, no. 11, pp. e0167492, 2016. DOI: 10.1371/journal.pone.0167492 
[13] Nabukeera-Barungi N., Elyanu P., Asire B., Katureebe C., Lukabwe I., Namusoke E., Musinguzi J., Atuyambe L., N. Tumwesigye, "Adherence to antiretroviral therapy and retention in care for adolescents living with HIV from 10 districts in Uganda," BMC Infectious Diseases, vol. 15, no. 1, pp. 1-10, 2015. DOI: 10.1186/s12879-015-1265-5

[14] Xu L., Munir K., Kanabkaew C., S. Le Coeur, "Factors influencing antiretroviral treatment suboptimal adherence among perinatally HIV-infected adolescents in Thailand," PloS one, vol. 12, no. 2, pp. e0172392, 2017. DOI: 10.1371/journal.pone.0172392

[15] Nabunya P., Bahar OS., Chen B., Dvalishvili D., Damulira C., FM. Ssewamala, "The role of family factors in antiretroviral therapy (ART) adherence self-efficacy among HIV-infected adolescents in southern Uganda," BMC Public Health, vol. 20, no. 1, pp. 1-9, 2020. DOI: 10.1016/j.conctc. 2019.100463

[16] Ioannides KL., Chapman J., Marukutira T., Tshume O., Anabwani G., Gross R., ED. Lowenthal, "Patterns of HIV treatment adherence do not differ between male and female adolescents in Botswana," AIDS and Behavior, vol. 21, no. 2, pp. 410-414, 2017. DOI: 10.1007/s10461-016-1530-7

[17] Lowenthal ED., Jibril HB., Sechele ML., Mathuba K., Tshume O., GM. Anabwani, "Disclosure of HIV status to HIV-infected children in a large African treatment center: Lessons learned in Botswana," Children and Youth Services Review, vol. 45, pp.143-149, 2014. DOI: 10.1016/j.childyouth.2014.03.031

[18] Martinez J., Harper G., Carleton RA, Hosek., S., Bojan K., Clum G., \& Ellen, and the Adolescent Medicine Trials Network, J, The impact of stigma on medication adherence among HIV-positive adolescent and young adult females and the moderating effects of coping and satisfaction with health care. AIDS patient care and STDs, vol. 26, no 2, pp, 108-115, 2012. DOI: 10.1089/apc.2011.0178

[19] Madiba, S., N Mohlabane, "Attendance of psychosocial teen clubs and self-reported antiretroviral medication adherence: a cross section study of adolescents with perinatal HIV in the Kingdom of Lesotho," AIMS Public
Health, vol. 8, no. 3, pp, 541-552, 2021. DOI: 10.3934/publichealth.2021044

[20] Madiba S., M. Mokgatle, "Fear of stigma, beliefs, and knowledge about HIV are barriers to early access to HIV testing and disclosure for perinatally infected children and adolescents in rural communities in South Africa," South African Family Practice, vol. 59, no. 5, pp. 175-181, 2017. DOI: $10.1080 / 20786190.2017 .1329489$

[21] Chesney MA., Ickovics J., Chambers D., Gifford A., Neidig J., Zwickl B., Wu AW., and Patient Care Committee \& Adherence Working Group of the Outcomes Committee of the Adult AIDS Clinical Trials Group (AACTG), 2000, "Self-reported adherence to antiretroviral medications among participants in HIV clinical trials: the AACTG adherence instruments," AIDS Care, vol. 12, no 3, pp. 255-266, 2000. DOI: 10.1080/09540120050042891

[22] Hudelson C., L. Cluver, "Factors associated with adherence to antiretroviral therapy among adolescents living with HIV/AIDS in low- and middle-income countries: a systematic review," AIDS Care, vol. 27, no. 7, pp. 805-816. 2015. DOI: 10.1080/09540121.2015.1011073

[23] Obua C., Kayiwa J., Waako P., Tomson G., Balidawa H., Chalker J., Ross-Degnan D., R. Wahlstrom, "Improving adherence to antiretroviral treatment in Uganda with a low-resource facility-based intervention," Global Health Action, vol. 7, no. 1, pp. 24198, 2014. DOI: 10.3402/gha.v7.24198

[24] Madiba S., U. Josiah, "Perceived Stigma and Fear of Unintended Disclosure are Barriers in Medication Adherence in Adolescents with Perinatal HIV in Botswana: A Qualitative Study," BioMed Research International, vol. 2021 pp. e9623159. 2019. DOI: 10.1155/2019/9623159

[25] Chikwari CD., Ferrand RA., V. Simms, "Association between self-reported adherence and HIV viral load suppression among older children and adolescents," Journal of Acquired Immune Deficiency Syndromes (1999), vol. 76, no. 3, pp. e87, 2017. DOI: 10.1097/QAI.00000000000015 01 